\title{
Vaccine Development Against Helminth Parasites
}

\author{
Phan Tran* \\ School of Science and Technology, University of New England, Australia
}

Received Date: July28, 2018; Published Date: August 16, 2018

*Corresponding author: Phan Tran, School of Science and Technology, University of New England, Armidale, NSW, Australia; Email: ptran4@myune.edu.au

\begin{abstract}
Helminth parasites represent a major obstacle for health and productivity in animals worldwide. The infection rate of parasitic diseases is responsible for vast economic losses in livestock production. Among various control methods, vaccination is considered as one of the best methods to reduce economic losses caused by parasitic diseases. However, some problems such as complicated characteristics of parasites and a high initial price of vaccine make this method limited. This paper reviews research mainstream on vaccines, control methods of parasites, introduction to the Trichostrongylus colubriformis parasite and vaccination, and challenges of vaccine development. Future research on vaccine development against helminth parasites is suggested.
\end{abstract}

\section{Introduction}

Parasite diseases are a main obstacle in inhibiting productivity of animals, thus reducing efficient livestock production. Both parasites, living inside the body and living on the body, are key agents for huge economic losses in animal production, especially cattle and sheep [1]. This fact provides a strong incentive for researchers to seek appropriate vaccines against parasites. Although development of vaccines against parasites faces challenges such as the complexity and diversity of parasites [2], research on anti-parasite vaccines has attracted much interest of researchers and scholars in medical sciences for the aim of searching the most effective way to treat animal diseases.

This paper aims to present a short review on

a) Current research mainstreams on vaccines;

b) Control methods of parasites;

c) An introduction to Trichostrongylus colubriformis parasite;

d) Vaccination including the development of immunity against parasite and the development of vaccines against Trichostrongylus colubriformis;

e) Challenges of vaccine development;

f) Suggestions on future research.

\section{Current Research Mainstreams}

It is widely recognized that there are two main aspects of current research mainstreams including the market for vaccines against parasites and the progress of parasite vaccines against helminth. At the first glance, the market for vaccines against parasites is enormous because there are more than 18 billion animals affected by parasites, however, until now very few effective vaccines are available to protect animals from helminth parasite infections [3]. Helminth parasites have reportedly influenced animal health and productivity for a long time, including tropical maladies caused by helminth worm parasites [2]. Treatment methods of parasites on livestock are dominated by drugs utilization [4]. According to Meeusen, et al. (As cited by Tak et al. [4]), this situation has changed by the demand of consumers for food free of chemicals. In today's vaccine market, although many types of vaccines have been developed, as indicated in Table 1, just a few vaccines can be commercialized due to a quantity of technical factors [1]. The types of vaccines in Table 1 could treat some specific parasites, for example, anaplaz can cure anaplasmosis which is a vector-borne, infectious blood disease in cattle caused by the rickettsia parasites. Another example is Toxovax that can treat toxoplasmosis disease caused by Toxoplasma gondii, an intracellular protozoan parasite that lives on sheep. However, these vaccines could not be effective for special parasites such as Trichostrongylus colubriformis. This leads to a growing demand for developing safe and effective vaccines consisting of anti-parasite commercial vaccines.

Table 1: Commercial vaccines in the market

\begin{tabular}{|c|c|}
\hline Disease, Parasite & Trade name \\
\hline Avian coccidiosis (Eimeria spp.) & $\begin{array}{c}\text { Paracox, Coccivax, } \\
\text { Livacox, Immucox }\end{array}$ \\
\hline Toxoplasmosis of sheep (Toxoplasma gondii) & Toxovax \\
\hline Giardiosis of dogs (Lamblia spp.) & GiardiaVax \\
\hline Anaplasmosis of cattle (Anaplas ma marginale) & Anaplaz \\
\hline Lungworn (Dictyocaulidae) & Huskvac, Dictol \\
\hline Taxas fever tick (Boophilus mi croplus) & TickGard, Gavac \\
\hline
\end{tabular}

Source: Adapted from Antiparasitics: Products and Markets by Tak et al. [4] 
The lack of progress in vaccine development against helminth parasites is another factor worth mentioning here. According to Hewitson \& Maizels [2], the reason for this is the complexity and diversity sourced from the helminth parasites. The helminths consist of different but related parasites, Nematoda and the Flatworm Platyhelminthes. The latter itself is separated into Cestodes (tapeworms) and Trematodes (flukes). These groups are classified into different branches, though they share similar patterns of transmission, infection and pathogenesis. However, in some cases of chronic helminth infection, it causes difficulty to eliminate the parasite from the body. Thus, it would lead to a challenge to develop a new vaccine against helminth parasites [7]. Supporting this view, Morrison \& Tomely [3] argued that a shortage of in vitro methods for the culture of parasites is also a significant constraint for the progress of vaccines. It can be seen that these problems could potentially provide an avenue for researchers to keep going on searching and developing appropriate vaccines against helminth parasites in order to improve economic profit in livestock production.

\section{Control Methods of Parasites}

There are four methods to control parasites including chemotherapy and chemoprophylaxis, integrated control, biological control and immunoprophylaxis are commonly used. To be more specific,

(i) Chemotherapy and chemoprophylaxis are considered as a control method against parasites. This method has long been used in the world. However, it faces risks to which the animals may be exposed to toxic from these two types [5]. In such a sense, this method is not popular today due to negative effects from residues and environmental issues [1];

(ii) The integrated control is a combination of biological, bio-technological and chemical control approach. This method aims to reduce chemical control agents to the lowest level [1]. Following Larsen [6], such an integrated approach is likely to obtain the most effective control because it can reduce infection levels and increase the immunity level;

(iii) Biological control is referred to as the use of one living organism to control a target parasite population and thus help to reduce the population growth of the latter [1]. According to Larsen [6], nematophagous fungi, for example Duddingtonia flagrans is very efficient to control gastrointestinal nematodes of livestock by lowering pasture infectivity;

(iv) Immunoprophylaxis is considered as the most promising way to eliminate infectious agents in livestock [1]. However, vaccines to control most of the parasitic infections of animals are not commercially available except for vaccine produced from Dictyocaulus viviparus [1,7]. Overall, these methods can be used to control parasites. However, depending on characteristics of each type of parasites, a combination of these methods is required to generate the best result of control.

\section{Trichostrongylus colubriformis Parasite}

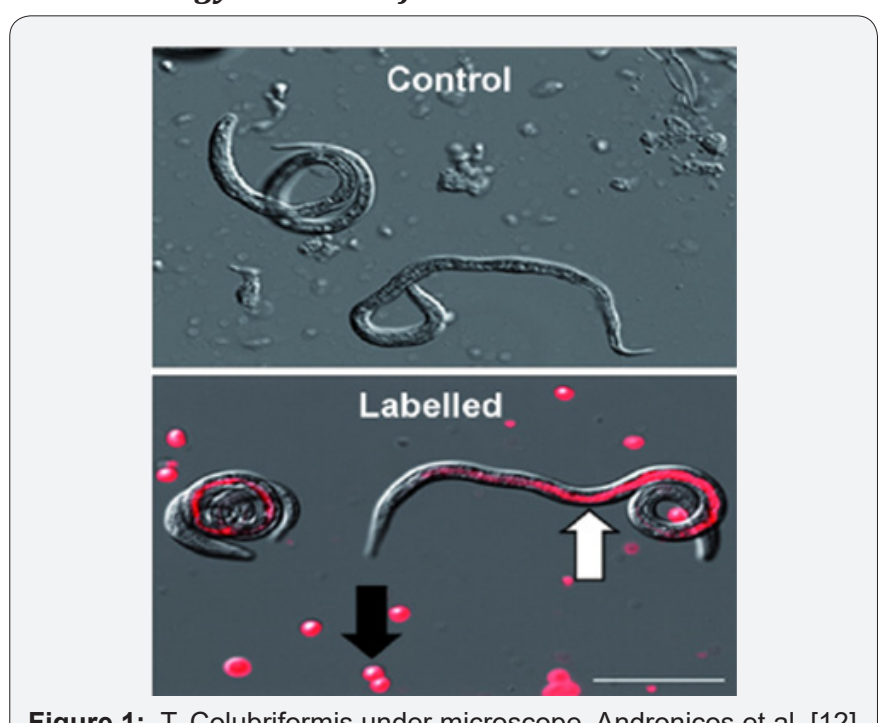

Figure 1: T. Colubriformis under microscope, Andronicos et al. [12]

In order to apply a proper control method for the helminth parasite like T. colubriformis and to develop an appropriate vaccine against this parasite, its characteristics and the rate of infection on human and animals in different countries and on livestock production in Australia should be perused. By definition, T. colubriformis is known as an intestinal parasite in animals with a wide-range distribution [8]. According to Phosuk at el. [9], there are 30 species of T. colubriformis affecting mammals and only 10 species influencing human. T. colubriformis is considered as the main zoonotic species causing human infection $[8,10]$. The infection of T. colubriformis is detected in eggs through experiments in the laboratory using fecal samples. Figure 1 displays the shape of T. colubriformis under microscope. Specifically, the upper image shows untreated or normal status of T. colubriformis parasite, so-called control, whereas the lower image showed treated status of T. colubriformis with colouring, marked as labelled. However, it is hard to distinguish between morphology (namely shape, composition and life environment) of T. colubriformis in eggs and other hookworm species because of their similar features [14]. The reason for human infections is the result from close contact with animals and this leads to symptoms such stomachaches, diarrhea and eosinophilia. Recently, T. colubriformis has been reported to have influence on human and animals in some countries such as Laos, Thailand, Iran, South Korea, China, the United States, and Australia $[8,11]$. This problem brings attention of researchers to develop appropriate vaccines to stop their influences and recover animal productivity and human health.

In Australia, the effects of T. colubriformis are mainly on animal health, namely sheep, thus these effects would cause a reduction in economic profit of sheep production. Recent work of Corvan, et al. [10] indicated that T. colubriformis can be treated successfully if T Helper Cell Type 2 (Th2) induced by gastrointestinal nematode (GIN) is utilized. However, the challenge faced with the GIN T. colubriformis parasite is that 
the protective Th2 responses of sheep are characterized by anti-parasite antibodies and eosinophilia $[10,12]$. In this sense, vaccine that might be developed is able to provide stimuli for the same immune response. However, Andronicos et al. [10] asserted that this approach has not implemented widely successful due to an inability of a killed vaccine to activate immune response that imitate accurately natural GIN infection. In short, T. colubriformis is one type of helminth parasites with differentiated characteristics, meaning that more requirements and conditions are necessary to develop appropriate vaccines to combat it successfully.

\section{Vaccination against Helminth Parasites}

The detail of vaccination is useful to show the development of immunity against parasites and that of vaccines against T.colubriformis. Vaccination has been widely known as the most popular solution to control parasite diseases, thus protect the most vulnerable animals in a herd for purpose of reducing the rate of infection in susceptible animals [1,3]. Regarding immunity against parasites, there are some differences between developing immunity against parasites with immunity against bacteria or virus. According to Tak et al. [4], the development of vaccines to control helminth parasites is enormously impacted by rapid development of the molecular biology in this era. However, helminth parasites have not only a complex developmental cycle in their host but also plenty of antigen components expressed at every stage. Therefore, against these organisms, vaccination can present a great deal of alternate targets [2]. As a consequence, vaccines designed efficiently need to be related to several inter disciplines. Parasitological techniques are the most important basic technique, which maintain the parasite cultures and supply ingredient for antigen purification as an element for developing a new effective vaccine [4]. Hence, there are not many commercial parasite vaccines on the market today.

Relating to the development of vaccines for T.colubriformis parasites, it can be observed that characteristics of T.colubriformis parasite are different from other ruminant nematode parasites, for example, adults are located in in the lumen of the small intestine. Therefore, failure for most recombinant protein vaccine against this parasite is common [2]. Emery, et al. [7] argued that a successful vaccine against nematodes may need to generate a series of local cellular and humoral responses. Hewitson \& Maizels [2] stated that "lambs are immunized with a combination of eight recombinant T. circumcincta". This means that a successful immunization can result in combining eight types of recombinant $T$. circumcincta. Although this result has been just successful in the laboratory, it shows a possible feasibility of recombinant vaccines against these parasites.

\section{Challenges of Vaccine Development}

The challenges of vaccine development are also important to discuss. These challenges can be listed as follows in three subcontents: what are difficulties for vaccine development, reasons for failure and specific obstacles for vaccine development against T.colubriformis. In more detail, the process of vaccines development against parasites is complicated and faces several challenges. Following Hewitson \& Maizels [2], key impediments in vaccines development consist of identifying the protective antigens, pinpointing the protective mechanism and providing an appropriate incentive to mobilize these pathways. Morrison \& Tomley [3] pointed out that the ability of many parasites to modulate host immune responses can lead to a consequence of parasite clearance delayed. These difficulties make many efforts of recent studies to develop vaccines for helminths unsuccessful.

Reasons for failure of a vaccine can come from different sources. According to Kebede, et al. [1], these reasons can be listed as follows. First, the vaccines may not be infective due to using the wrong antigens or insufficient antigens and even unsatisfactory administration. For instance, if animals are vaccinated in the incubation period, the vaccine may not be effective for the course of disease. Second, when the normal immune response is inhibited, the failure of vaccine happens. Some stress like fatigue, malnourishment, and pregnancy may reduce normal immune response. Emery, et al. [7] revealed that enteric immune response depends on both route of delivery and adjuvant. So, this factor needs to be concerned in the process of vaccine development. Finally, Kebede, et al. [1] showed that vaccine efficacy is an enhancer to make the failure of a vaccine reduced. In doing so, antigen cells must be activated to process efficiently antigens and release appropriately interleukins. Similarly, T and B cells (so-called lymphocytes that develop in bone marrows similar to all blood cells) should be stimulated to yield a large quantity of memory cells. Moreover, the antigen must persist in suitable sites. Once three contents are treated efficiently, success of vaccine development may be recognised.

Regarding challenges of developing vaccines for T.colubriformis, some conditions need to be satisfied to achieve the goal of vaccination. According to Hewistson \& Maizels [2], the mode of immune responsiveness must be correct, in which the Th2 pathway is present in most helminth settings. This is followed by the type of response through signals from innate cells and tissues that is vitally determined to provide the appropriate stimulation of the innate compartment for the design of new vaccines. Andronicos, et al. [13] found that the mode of host resistances of helminth parasites including T.colubriformis is different though some similarities of host sensibility is present. In addition, Emery, et al. [7] illustrated that local responses should be effective against nematodes like T.colubriformis. These factors should be considered for the purpose of producing a successful vaccine.

\section{Suggestions on Future Research}

Generally, it is known that this process is much more difficult. This is because T. colubriformis affects both human and animals and the rate of its infection is reportedly enormous over the world $[8,14]$. It is noted that the difficulty of vaccine development has not included high costs of vaccination in the initial phase [14]. 
In spite of these challenges, research on vaccines for this type of parasite has attracted much interest of researchers and scholars, for example Goodswen, et al. [15] suggested an application of reverse vaccinology against parasites that is worth of concern. On top of this, to be able to seek an effective solution for vaccine development against this parasite, the following objectives should aim to first

a) Define the physiochemical characteristics that facilitate the development of T. colubriformis from L3 larvae into sexually mature adults,

b) Define antigens from the L3, L4 and adult stages of $T$. colubriformis that react to antibodies from sheep immunologically resistant to $\mathrm{T}$. colubriformis,

c) Use recombinant DNA technology to generate $T$. colubriformis antigen for incorporation to an experimental vaccine, and

d) Test the vaccine in a $T$. colubriformis permissive laboratory animal model (rabbit or guinea pig).

Once these objectives can be done, the probability of developing a successful vaccine may be higher, and then the opportunity of developing a commercial vaccine is greater. In order words, social wellbeing can be improved including an increase in economic profit of animal production.

\section{Conclusion}

This paper provides a brief review on vaccine development against helminth parasites in general and T. colubriformis in particular. The distribution of the infection of these parasites is worldwide, causing huge influence on human health and livestock production. This leads to a critical question of how to combat these parasites successfully and that is still a challenge for scientists. Indeed, the development of vaccine against parasites is a potential solution to overcome this problem; however, there are several things to be considered in the process of designing a new vaccine. Although it is not easy to find and develop an effective vaccine, it seems that scientists never give up. Instead, they put more efforts into research on vaccine development in order to improve animal productivity and human welfare.

\section{References}

1. Kebede B, Sori T, Kumssa B (2016) Review on Current Status of Vaccines against Parasitic Diseases of Animals. Journal Veterinary Science Technology 7(3): 1-8.
2. Hewitson JP, Maizels RM (2014) Vaccination against helminth parasite infections. Expert Rev Vaccines 13(4): 473-487.

3. Morrison WI, Tomley F (2016) Development of vaccines for parasitic diseases of animals: Challenges and opportunities. Parasite Immunology 38(12): 707-708.

4. Tak IR, Dar JS, Dar SA, Ganai BA, Chishti MZ, et al. (2015) A comparative analysis of various antigenic proteins found in Haemonchus contortus-A review. Molecular Biology 49(6): 789-795.

5. Leach TM, Roberts CJ (1980) Present status of chemotherapy and chemoprophylaxis of animal trypanosomiasis in the eastern hemisphere. Pharmacology \& therapeutics 13(1): 91-147.

6. Larsen, M. (1999). Biological control of helminths. International Journal for Parasitology, 29(1): 139-146.

7. Emery DL, McClure SJ, Wagland BM (1993) Production of vaccines against gastrointestinal nematodes of livestock. Immunology \& Cell Biology 71(5): 463-472.

8. Sato M, Yoonuan T, Sanguankiat S, Nuamtanong S, Pongvongsa T, et al. (2011) Human Trichostrongylus colubriformis infection in a rural village in Laos. The American journal of tropical medicine and hygiene 84(1): 52-54.

9. Phosuk I, Intapan PM, Sanpool O, JanwanP, Thanchomnang T, et al. (2013) Molecular evidence of Trichostrongylus colubriformis and Trichostrongylus axei infections in humans from Thailand and Lao PDR. American J Trop Med and Hyg 89(2): 376-379.

10. Corvan SM, Agnew L, Andronicos NM (2015) Trichostrongylus colubriformis induces IgE-independent CD13, CD164 and CD203c mediated activation of basophils in an in vitro intestinal epithelial cell co-culture model. Vet Parasitol 207(3-4): 285-296.

11. Buonfrate D, Angheben A, Gobbi F, Mistretta M, Degani M, et al. (2017) Four clusters of Trichostrongylus infection diagnosed in a single center, in Italy. Infection 45(2): 233-236.

12. Andronicos NM, McNally J, Kotze AC, Hunt PW, Ingham A (2012) Trichostrongylus colubriformis larvae induce necrosis and release of IL33 from intestinal epithelial cells in vitro: implications for gastrointestinal nematode vaccine design. Int J for Parasitol 42(3): 295-304.

13. Andronicos N, Hunt P, Windon R (2010) Expression of genes in gastrointestinal and lymphatic tissues during parasite infection in sheep genetically resistant or susceptible to Trichostrongylus colubriformis and Haemonchus contortus. International Journal for Parasitology 40(4): 417-429.

14. Patra G, Kumar A, Ghosh S, Lalnunpuia C, Bachan M, et al. (2017) Vaccines against protozoan parasites of veterinary importance: A review. Journal of Entomology and Zoology Studies 5(6): 1016-1021.

15. Goodswen SJ, Kennedy PJ, Ellis JT (2017) On the application of reverse vaccinology to parasitic diseases: a perspective on feature selection and ranking of vaccine candidates. Int J Parasitol. 47(12): 779-790. 
This work is licensed under Creative Commons Attribution 4.0 License

DOI: $10.19080 /$ NAPDD.2018.04.555629

\section{Your next submission with Juniper Publishers} will reach you the below assets

- Quality Editorial service

- Swift Peer Review

- Reprints availability

- E-prints Service

- Manuscript Podcast for convenient understanding

- Global attainment for your research

- Manuscript accessibility in different formats

( Pdf, E-pub, Full Text, Audio)

- Unceasing customer service

Track the below URL for one-step submission https://juniperpublishers.com/online-submission.php 